\title{
A NEW MECHANISM FOR DATA VISUALIZATION WITH TSK-TYPE PREPROCESSED COLLABORATIVE FUZZY RULE BASED SYSTEM
}

\author{
Mukesh Prasad ${ }^{1}$, Yu-Ting Liu ${ }^{2,3}$, Dong-Lin $\mathrm{Li}^{2}$, Chin-Teng Lin ${ }^{2,3}$, \\ Rajiv Ratn $\mathrm{Shah}^{4}$, Om Prakash Kaiwartya ${ }^{5}$ \\ ${ }^{1}$ Department of Computer Science, National Chiao Tung University, Hsinchu, Taiwan \\ ${ }^{2}$ Department of Electrical Engineering, National Chiao Tung University, Hsinchu, Taiwan \\ ${ }^{3}$ Faculty of Engineering and Information Technology, University of Technology Sydney, Sydney, Australia \\ ${ }^{4}$ School of Computing, National University of Singapore, Singapore \\ ${ }^{5}$ Faculty of Computing, Universiti Teknologi Malaysia, Skudai Johor, Malaysia
}

\begin{abstract}
A novel data knowledge representation with the combination of structure learning ability of preprocessed collaborative fuzzy clustering and fuzzy expert knowledge of TakagiSugeno-Kang type model is presented in this paper. The proposed method divides a huge dataset into two or more subsets of dataset. The subsets of dataset interact with each other through a collaborative mechanism in order to find some similar properties within eachother. The proposed method is useful in dealing with big data issues since it divides a huge dataset into subsets of dataset and finds common features among the subsets. The salient feature of the proposed method is that it uses a small subset of dataset and some common features instead of using the entire dataset and all the features. Before interactions among subsets of the dataset, the proposed method applies a mapping technique for granules of data and centroid of clusters. The proposed method uses information of only half or less/more than the half of the data patterns for the training process, and it provides an accurate and robust model, whereas the other existing methods use the entire information of the data patterns. Simulation results show the proposed method performs better than existing methods on some benchmark problems.
\end{abstract}

Keywords: fuzzy interference system, collaborative clustering, fuzzy logic, big data, data visualization

\section{Introduction}

Fuzzy logic has been applied successfully in various applications such as vehicle control, agricultural engineering, astronomy, chemistry, geology, image analysis, medical diagnosis, shape analysis and target recognition, consumer products, and control of manufacturing systems etc. Fuzzy logic is one of the most widely use technologies for developing complex feedback control systems for inexpensive processors due to its simplicity and ease in adopting to any environment. Fuzzy logic has the capability of providing a good and robust decision from incomplete information or less available knowledge with perfectly addressing as it acts like human decision making, whereas other techniques 
such as, linear control design and purely logicbased models, require exact equations to model real-world behaviors. There are basically three types of fuzzy inference systems: Mamdani type, Takagi-Sugeno-Kang (TSK) type and logical type fuzzy inference system models. All of them contain the expert knowledge of fuzzy logic with their own problem solving capability. Traditionally, Mamdani model [1] is widely accepted as a manner to build expert knowledge. It allows designers to describe the expertise in an intuitive way. However, Mamdani-type fuzzy inference system consumes large amount of effort for computation. On the other hand, Sugeno method [2], which is known as Takagi-Sugeno-Kang (TSK) fuzzy model, is computationally effective and works well with optimization and adaptive techniques. Therefore, TSK type model is very attractive to researchers to solve control problems, particularly for dynamics nonlinear systems. Logical-type represented by an Simplication would be preferred for classification problems logical-type systems or other fuzzy implications [3, 4]. This paper's simulation results are based on TSK type model.

Fuzzy rule based modeling system or fuzzy control rules first extract expert knowledge from its knowledge bases, and then build expert systems. Fuzzy expert system is a combination of rules and membership function, which is generated by fuzzy c-means (FCM) clustering or some other clustering methodology [12-19]. The success of the Fuzzy expert system depends on the quality of acquired knowledge. Fuzzy control rule can be expressed in following way:

a) IF temperature is very low and pressure is normal, THEN heat change would be slightly positive.

b) IF it is raining and wind is fast and dark outside, THEN take an umbrella and a torch.

However, in many real situations it is not a good way to combine expert knowledge, and it is necessary to resort the learning system to acquire knowledge. There exist several methods for the learning of fuzzy rules. An example as follows: suppose we have variety of information with the same patterns from different field applications. To get a comprehensive study of these varieties of informa- tion, knowledge based clustering [7] and collaborative clustering between datasets is recommended. Pedrycz $[8,9]$ introduced a collaborative clustering to solve the problem when some data cannot acquire directly from the dataset due to data confidentiality. In this kind of clustering algorithm, several subsets of patterns can be processed together with an objective of finding a common structure that is shared by all of them. In this study, preprocessed collaborative fuzzy clustering (PCFC) technique is applied to generate a number of rules to calculate the membership function. PCFC has the ability to extract good knowledge from the given unidentified information. We combined PCFC rule learning mechanism with TSK type model, which helps modeling system to design an accurate and robust model.

The rest of the paper is organized as follows: Section 2 gives introduction of FCM, PCFC, TSK type FIS, the proposed system, and flow diagram of the proposed system. Section 3 shows the simulation results on two nonlinear dynamic system, and compares the proposed method with the Matlab based Genfis2 method [11]. Finally, the conclusions are covered in Section 4.

\section{Basic Procedure and Proposed Algorithm}

\subsection{Fuzzy C-Means Clustering}

Fuzzy C-Means (FCM) $[5,6]$ is one of the most common unsupervised clustering algorithms, which is originally described first by Bezdek in 1973. Variants of FCM [21-24] have been described with modified definitions for the norm and prototypes of the cluster centroids [26-28]. FCM clusters each data point to one or more clusters, and partitions a set of data $x_{i} \in R^{d}, i=1,2, \ldots, N$ into a certain number $c$ of fuzzy clusters by minimizing the following cost function

$$
J_{m}=\sum_{i=1}^{N} \sum_{j=1}^{c} u_{i j}^{m}\left\|x_{i}-v_{j}\right\|^{2},
$$

where $\mathrm{m}$ is any real number greater than $1, u_{i j}$ is the degree of membership of $x_{i}$ in the cluster $j, x$ is the $i$-th data point of $d$-dimension data, $v_{j}$ is the $d$ dimension of the cluster $j$, and $\|*\|$ is any norm expressing the similarity between any measured data and the center. 


\subsection{Procedure of FCM}

1. Set up a value of c (number of cluster);

2. Select initial cluster prototype $V_{1}, V_{2}, \ldots \ldots, V_{c}$ from $X_{i}, i=1,2, \ldots, N$;

3. Compute the distance $\left\|X_{i}-V_{j}\right\|$ between objects and prototypes;

4. Compute the elements of the fuzzy partition ma$\operatorname{trix}(i=1,2, \ldots, N ; j=1,2, \ldots, c)$

$$
u_{i j}=\left[\sum_{l=1}^{c}\left(\frac{\left\|x_{i}-v_{j}\right\|}{\left\|x_{i}-v_{l}\right\|}\right)\right]^{-1}
$$

5. Compute the cluster prototypes $(j=1,2, \ldots, c)$

$$
V_{j}=\frac{\sum_{i=1}^{N} u_{i j}^{2} x_{i}}{\sum_{i=1}^{N} u_{i j}^{2}}
$$

6. Stop if the convergence is attained or the number of iterations exceeds a given limit. Otherwise, go to step 3.

\subsection{Takagi-Sugeno-Kang Fuzzy Inference System}

TSK fuzzy inference model proposed by Takagi, Sugeno, and Kang [2, 20], has been widely used in control and fuzzy modeling. The key idea of TKS is to divide input space into several fuzzy regions, and approximate each region by a simple model. All the systems can be regarded as a combination of a series of simple models. In general, TSK can be represented in mathematical form as

$R_{i}: \operatorname{IF} x_{1}(t)=A_{1, j}^{(i)}$ AND $\cdots$ AND $x_{n}(t)=A_{n, j}^{(i)}$,

THEN $y_{s}^{(i)}=f_{s}^{(i)}(x(t))$,

where $R_{i}$ is $i$-th rule in TSK model, $t$ denotes a sampling instant, and $X_{s}(t)=\left[x_{1}(t), \ldots, x_{s}(t)\right]^{T}$ is input vector. Each $x_{k} \in R$, where $k=1, \ldots, s, A_{k, j}^{(i)}$ the $j$ fuzzy set characterized by the $i$-th rule corresponding to the input $x_{k}, y_{s}$ is the output of overall model, and $f_{s}(X(t))$ is a first-order polynomial, can be computed as

$$
f_{s}\left(X(t), b^{i}\right)=\frac{\sum_{k=1}^{R_{i}} a_{s}^{(i)} f^{(i)}\left(x_{1}, \ldots, x_{s}\right)}{\sum_{i=1}^{R_{i}} \alpha_{s}^{(i)}}
$$

$R_{i}$ is the total number of rules, and $\alpha_{s}^{(i)}$ is the degree of matching between the $i$-th fuzzy and the $s$-th sample. $f^{(i)}\left(x_{1}, \ldots, x_{s}\right)$ can be represented as

$$
b_{0, s}^{i}+b_{1, s}^{i} x_{1}(t)+\ldots+b_{s-1, s}^{i} x_{s}(t),
$$

where $b_{k, s}^{i} \in R$. By above definition, $R_{i}=2$ and

$$
\alpha_{s}^{(i)}=\min \left\{\mu_{A_{1, j}^{(i)}} x_{1}^{\prime}, \mu_{A_{2, j}^{(i)}} x_{2}^{\prime}\right\}
$$

Figure 1 shows the computation process of TSK inference model. Ting and Quek [21] demonstrated a more complicated TSK inference model. Typical cost function $J_{T S K}$ measures how the TSK model approximates the real problem. The $J_{T S K}$ can be expressed as

$$
J_{T S K}=\sum_{t=1}^{N} D^{2}(t)
$$

where $D(t)$ is the difference of output between the real system and the identified model, and $N$ is the number of training samples.

A generic TSK inference model can be divided into four parts.

1. Partition the input space into $r$ inference rules.

2. Identify the structure of each IF part.

3. Identify the constitution of each THEN part.

4. Calculate the predicted value.

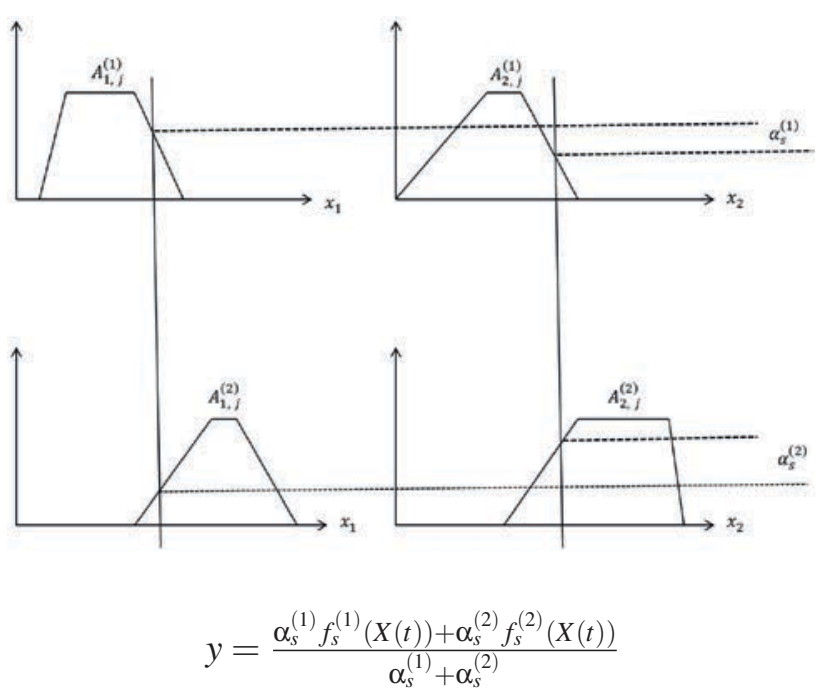

Figure 1. TSK inference model 


\subsection{Preprocessed Collaborative Fuzzy Clustering}

Pedrycz [8] introduced a collaborative fuzzy clustering (CFC) to find the consistency between two or more datasets. Its variants: horizontal [10] and vertical collaboration fuzzy clustering [25], also exist. Figure 2 and 3 show the general scheme of horizontal collaborative clustering and vertical collaborative clustering, respectively. The communication bridge in horizontal clustering is based on through the partition matrix, where data is deal with same pattern and different features spaces. The confidentiality of data is kept by operating on resulting information granules (partition matrices) instead on individual patterns due to the granularity of data is far different from the original data. The communication bridge in vertical clustering at the level of the prototypes (high-level representatives of data), where data is deal with different pattern but the same feature space. The confidentiality requirement of data is kept due to aggregate nature of the prototypes. Figure 4 shows the connections of matrices in order to accomplish the collaboration between the subsets of the database. Prototype and partition matrices bring the way of structural findings at the each dataset.

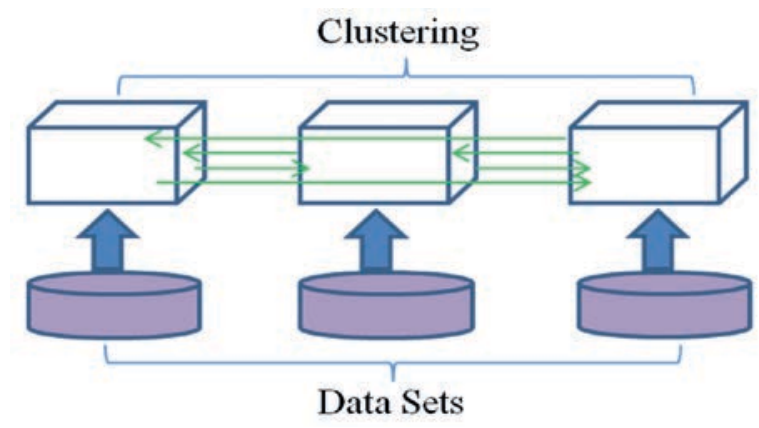

Figure 2. A General scheme of horizontal clustering

The minimization of objective function of CFC is defined as Eq. (8), where $\beta$ is a user defined parameter based on datasets $(\beta>0), \beta[l, m]$ denotes the collaborative coefficient with collaborative effect on dataset $l$ through $m, c$ is a number of cluster, $l=1,2, \ldots, P$ is a number of datasets, $N$ is the number of patterns in the dataset, $u$ represents the partition matrix, $n$ is a number of features, and $d$ is an Euclidean distance between patterns and prototypes.

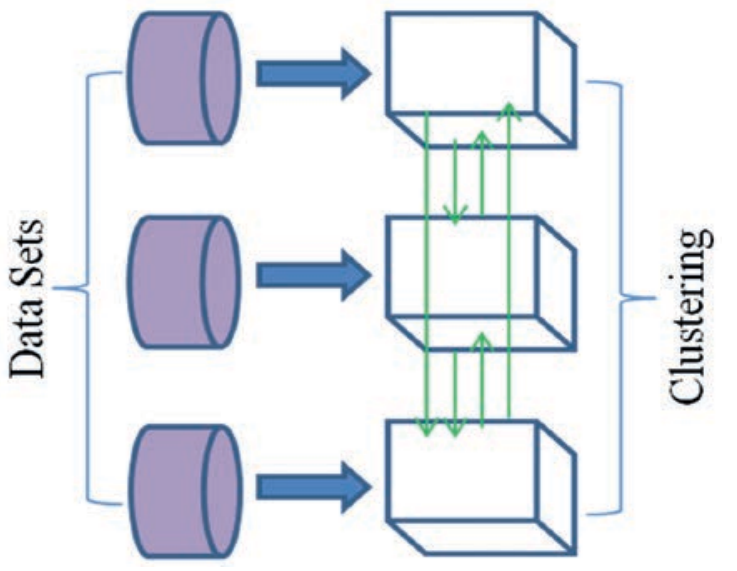

Figure 3. A General scheme of vertical clustering

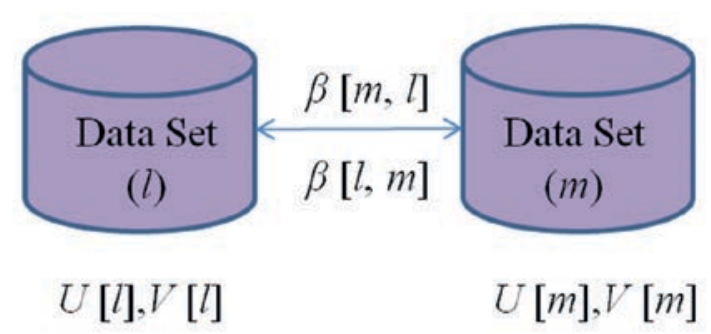

Figure 4. Collaborative clustering scheme for two dataset

Preprocessed collaborative fuzzy clustering (PCFC) proposed by Prasad [29] is a mapping mechanism for prototype and partition matrix before collaboration phase. Since direct subtraction of $u_{i k}[l]$ and $u_{i k}[\mathrm{~m}]$ may lose the meaning of difference between two membership degrees $u_{i k}[l]$ and $u_{i k}[\mathrm{~m}]$ under different partition matrices of one pattern $X_{k}$ to the same cluster, we have to find a constructive approach of the preprocessing in order to rearrange the rows order of $u_{i k}[l]$ corresponding to the rows order of $u_{i k}[\mathrm{~m}]$ in a rational way. The match rows pair is determined by

$$
r=\arg \min _{j=1,2 \ldots, c} \sum_{i=1}^{n}\left(v_{k i}[l]-v_{j i}[m]\right)^{2},
$$

where $n$ is the number of features. The k-th row of $v[l]$ and the r-th row of $v[m]$ are considered to be matched row pair $(k=1,2, \ldots, c)$. Similarly, this value is updated with $u_{i k}[l]$ and $u_{i k}[m]$.

The optimization task as shown in Eq. (8) is divided into two main parts those determine the partition matrix $U[l]$, and $v_{1}[l], v_{2}[l], \ldots, v_{c}[l]$. These determination problems are calculated sep- 


$$
\begin{gathered}
Q[l]=\sum_{i=1}^{N} \sum_{j=1}^{c} u_{i j}^{2}[l] d_{i j}^{2}[l]+\sum_{\substack{m=1 \\
m \neq l}}^{p} \beta[l, m] \sum_{i=1}^{N} \sum_{j=1}^{n}\left\{u_{i j}[l]-u_{i j}[m]\right\}^{2} d_{i j}^{2}[l] \\
\tilde{Q}[l]=\sum_{i=1}^{N} \sum_{j=1}^{c} u_{i j}^{2}[l] d_{i j}^{2}[l]+\sum_{\substack{m=1 \\
m \neq l}}^{p} \beta[l, m] \sum_{i=1}^{N} \sum_{j=1}^{c}\left\{u_{i j}[l]-u_{i j}[m]\right\}^{2} d_{i j}^{2}[l]-\lambda\left(\sum_{i=1}^{c} u_{i j}[l]-1\right)
\end{gathered}
$$

arately for each of the collaborating subsets of patterns. The Lagrange multipliers technique is used to determine the partition matrix in order to make constraint-free optimization. This takes us to solve the new objective function $\tilde{Q}[l]$ : Eq. (10), where $\lambda$ represents a Lagrange multiplier. The important conditions lead us to the local minimum of $\tilde{Q}[l]$ which are as follows:

$$
\frac{\partial \bar{Q}[l]}{\partial u_{s t}[l]}=0 ; \frac{\partial \bar{Q}[l]}{\partial \lambda}=0
$$

where $s=1,2, \ldots, c$ and $t=1,2, \ldots, N$. The derivative computed with respect to the partition matrix as follows: Eq. (12).

In other words, the partition matrix is calculated as:

$$
\begin{aligned}
& \lambda+2 d_{s t}^{2}[l] \sum_{m=1}^{p} \beta[l, m] u_{s t}[m] \\
& u_{s t}[l]=\frac{m \neq l}{2+\left(d_{s t}^{2}[l]+d_{s t}^{2}[l] \sum_{\substack{m=1 \\
m \neq l}}^{p} \beta[l, m]\right)},
\end{aligned}
$$

Now, we can shorten the Eq. (13) by introducing the following shorthand notation:

$$
\begin{gathered}
\varphi_{s t}[l]=\sum_{\substack{m=1 \\
m \neq l}}^{p} \beta[l, m] u_{s t}[m], \\
\psi[l]=\sum_{\substack{m=1 \\
m \neq l}}^{p} \beta[l, m] .
\end{gathered}
$$

Eq. (13) is expressed in the form of the expression as shown in Eq. (16) by the constraint imposed on the membership values $\sum_{i=1}^{c} u_{i j}[l]=1$.

$$
\sum_{i=1}^{c} \frac{\lambda+2 d_{i j}^{2}[l] \varphi_{i j}[l]}{2 d_{s t}^{2}[l](1+\psi[l])}=1 .
$$

Next, the Lagrange multiplier determines in the form as follows:

$$
\lambda=2 \frac{1-\frac{1}{1+\psi[l]} \sum_{i=1}^{c} \varphi_{i j}[l]}{\sum_{i=1}^{c} \frac{1}{d_{i j}^{2}[l]}}(1+\psi[l]) .
$$

Putting this multiplier into the partition matrix formula as shown in Eq. (13), this gives the final expression as follows:

$$
u_{s t}[l]=\frac{\varphi_{s t}[l]}{1+\psi[l]}+\frac{1}{\sum_{j=1}^{c} \frac{d_{s t}^{2}[l]}{d_{j t}^{2}[l]}}\left[1-\sum_{j=1}^{c} \frac{\varphi_{j t}[l]}{1+\psi[l]}\right]
$$

For the calculations of the prototypes the Euclidean distance is considered between the patterns and the prototypes. The necessary condition for minimizing the objective function is of the form $\Delta v[l] Q[l]=0$. Now the objective function as shown in Eq. (19) with distance function is expressed as follow: Eq. (19).

The patterns in this expression as shown in Eq. (19) come from the $l$-th data set. Computing the derivative of $Q[l]$ with respect to $v_{s t}[l]$ and setting it to 0 , we obtain Eq. (20).

The final expression for the prototypes after some grouping of the terms is as follows: Eq. (21)

Preprocessed collaborative fuzzy clustering partitioning is carried out through an iterative optimization of the objective function as shown in Eq. (8) with an update of partition matrix $U[l]$ and the prototype $v_{i}[l]$ as shown in Eq. (18) and (21) respectively. 


$$
\begin{gathered}
\frac{\partial \bar{Q}[l]}{\partial u_{s t}[l]}=2 u_{s t}[l] d_{s t}^{2}[l]+2 \sum_{\substack{m=1 \\
m \neq l}}^{p} \beta[l, m]\left(u_{s t}[l]-u_{s t}[m]\right) d_{s t}^{2}[l]-\lambda=0 \\
Q[l]=\sum_{i=1}^{N} \sum_{j=1}^{c} u_{i j}^{2}[l] \sum_{k=1}^{n[l]}\left(x_{i k}-v_{j k}[l]\right)^{2}+\sum_{\substack{m=1 \\
m \neq l}}^{p} \beta[l, m] \sum_{i=1}^{N} \sum_{j=1}^{c}\left\{u_{i j}[l]-u_{i j}[m]\right\}^{2} \sum_{k=1}^{n[l]}\left(x_{i k}-v_{j k}[l]\right)^{2}
\end{gathered}
$$

\subsection{Procedure for PCFC}

Based on the above discussions and the results, PCFC adds one more phase called phase II for mapping procedure before collaboration process and present the refined algorithm as follows:

1. Given: subsets of patterns $X_{1}, X_{2}, \ldots, X_{p}$,

2. Select: distance function, number of clusters (c), termination condition, and collaboration coefficient $\beta[l, m]$,

3. Compute: initiate randomly all partition matrices $u[1], u[2], \ldots u[p]$

- Phase I For each data

Repeat

Compute prototype $\left\{v_{j}[l], j=1,2, \ldots, c\right.$ and partition matrices $u[l]$ for all subsets of patterns\}

Until a termination condition has been satisfied

- Phase II Choose an approach for the preprocessing on cluster prototype and its corresponding partition matrices to adjust row order by using (9).

\section{- Phase III Repeat}

For the matrix of collaborative links $\beta[l, m]$

Compute, prototype $v_{j}[l]$ and partition matrices $u[l]$ by using Eq. (10) and (13)

Until a termination condition has been satisfied

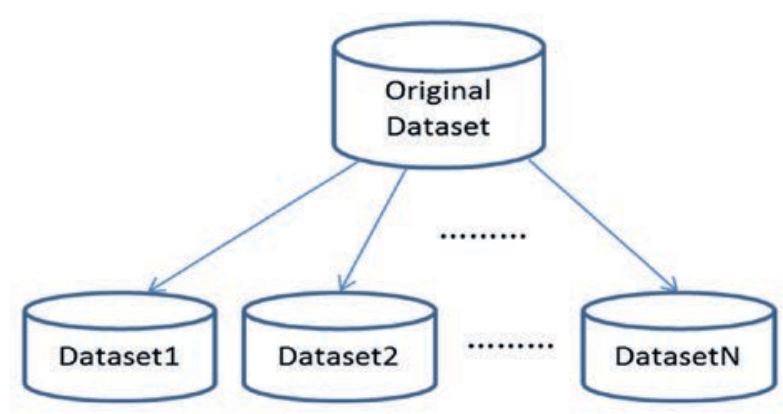

Figure 5. Division of an original dataset into $N$ different datasets

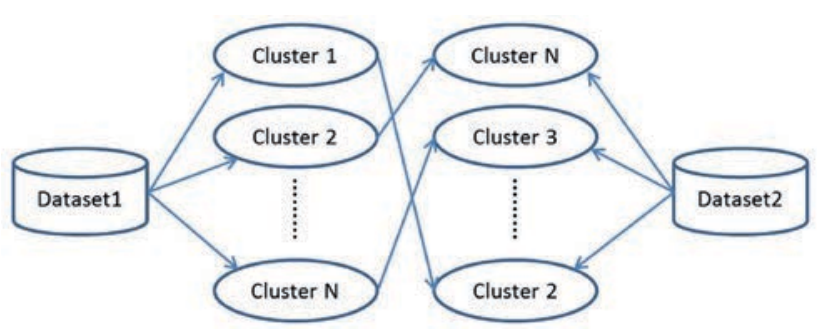

Figure 6. Representation of $N$ classes for dataset 1 and dataset 2 after FCM

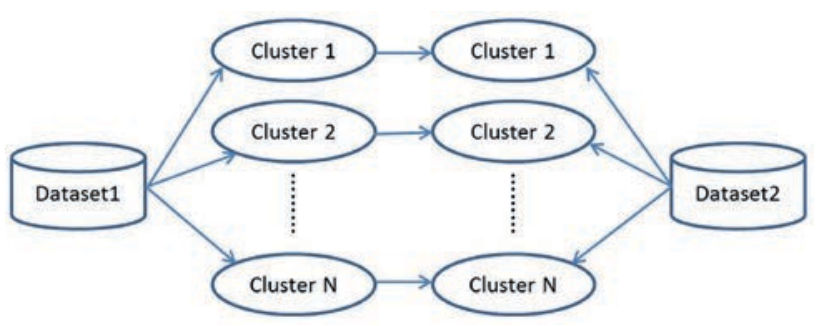

Figure 7. Representation of $N$ classes for dataset1 and dataset 2 after preprocessing

Figure 5 shows a general way of dividing a huge dataset into $\mathrm{n}$ numbers of equal data sites. Figure 6 shows the representation of $N$ number of 


$$
\frac{\partial Q[l]}{\partial v_{s t}[l]}=-2 \sum_{k=1}^{N} u_{s k}^{2}[l]\left(x_{k t}-v_{s t}[l]\right)-2 \sum_{k=1}^{N} \sum_{\substack{m=1 \\ m \neq l}}^{p} \beta[l, m]\left(u_{s k}[l]-u_{s k}[l]\right)^{2}\left(x_{k t}-v_{s t}[l]\right)=0
$$

$$
\begin{aligned}
& \sum_{k=1}^{N} u_{s k}^{2}[l] x_{k t}[l]+\sum_{m=1}^{p} \beta[l, m] \sum_{k=1}^{N}\left(u_{s k}[l]-u_{s k}[m]\right)^{2} x_{k t}[l] \\
& v_{s t}[l]=\frac{m \neq l}{\sum_{k=1}^{N} u_{s k}^{2}[l]+\sum_{\substack{m=1 \\
m \neq l}}^{p} \beta[l, m] \sum_{k=1}^{N}\left(u_{s k}[l]-u_{s k}[m]\right)^{2}}
\end{aligned}
$$

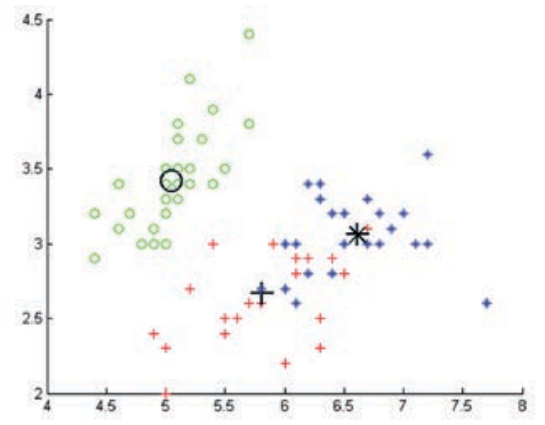

(a) Clustered feature vectors of dataset 1 based on FCM

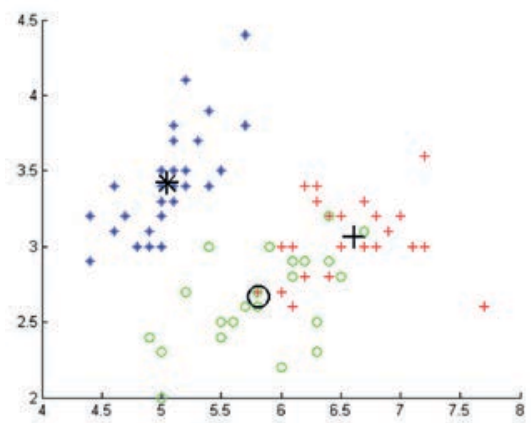

(c) Clustered feature vectors of dataset 1 based on FCM after mapping

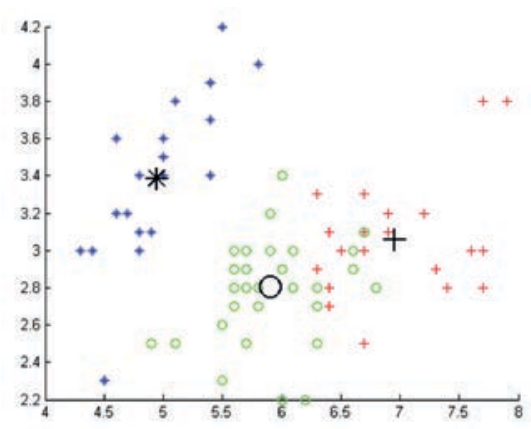

(b) Clustered feature vectors of dataset 2 based on FCM

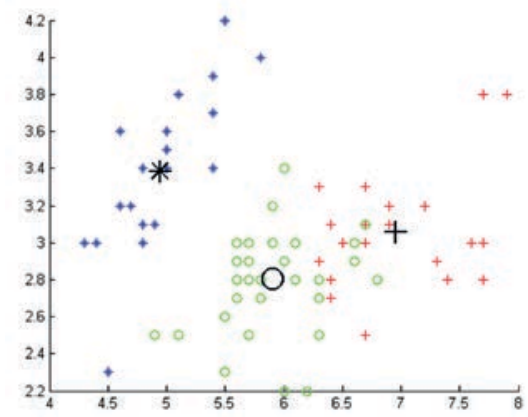

(d) Clustered feature vectors of dataset2 of FCM after mapping

Figure 8. Clustered feature vectors of dataset1 and dataset 2 


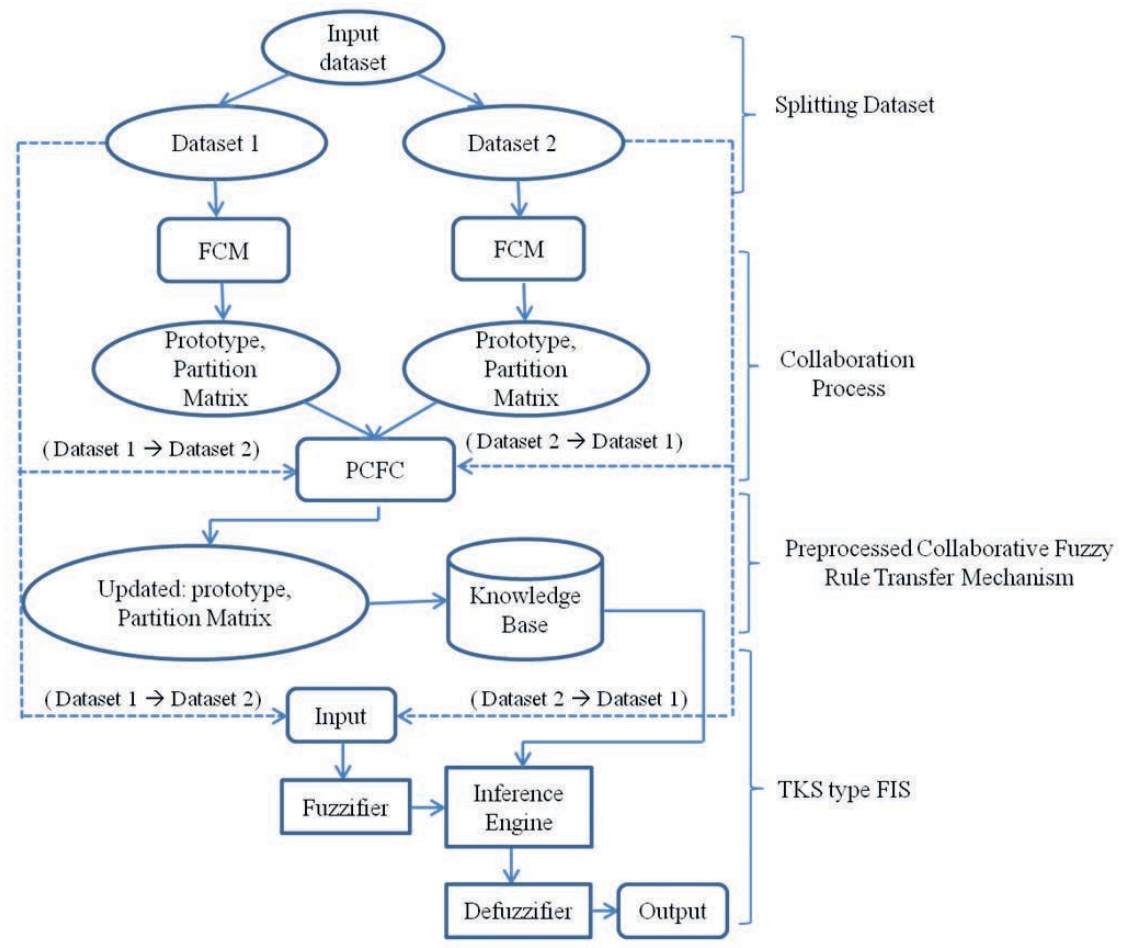

Figure 9. Architecture of proposed FIS with CFCM model

classes for two datasets: dataset 1 and dataset2, after FCM. Here it can easily visualize how rows pair are mismatched and the mismatch could lead the system in a different direction for analyzing the data. Figure 7 shows the correctness of $N$ number of classes matching of dataset 1 and dataset 2 after applying the mapping mechanism for prototypes and partition matrices. In order to verify the mapping mechanism, the proposed method has used the paradigm of three classes, and then divided them equally into two subsets of dataset: dataset 1 and dataset 2 . Figures $8(a)$ and $8(\mathrm{~b})$ are clustered feature vectors of dataset 1 and dataset 2 , respectively. As it can be seen, in Figures 8(a) and 8(b), the first cluster (green color) of dataset 1 matches with the second cluster of dataset 2 , the second cluster (red color) of dataset 1 matches with the third cluster of dataset 2 , and the third cluster (blue color) of dataset 1 matches with the first cluster of dataset 2 which are totally mismatched with each other. Figures $8(\mathrm{c})$ and $8(\mathrm{~d})$ show the plotting results after the mapping mechanism, where the effect of centroid mapping for prototype and row order mapping with the partition matrix can be seen. Now, we can easily take the difference(s) between rows of dataset 1 and dataset 2 , and easily do mapping between them.

\subsection{Architecture of the Proposed Model}

The proposed method, shown in Figure 9, combines the reasoning strengths of TSK type fuzzy inference system with the knowledge representation ability of mapped collaborative fuzzy cluster, and gives a robust and reliable modeling system. Firstly, the given input data is divided into two or more equally sub groups of dataset, and FCM is applied on each sub-groups of dataset separately to calculate prototypes and partition matrix for each dataset. Secondly, PCFC updates all partition matrix and prototype by collaborating each of them and gets a common feature among them, and provides these features to the knowledge based sub system of fuzzy inference system. Thirdly, the inference engine uses the knowledge from the knowledge based sub system along with fuzzier information of given dataset. Instead of providing the entire data patterns, the proposed method just uses half of the data patterns for further modeling process, while other methods use the entire data patterns. By using just half of the data patterns, the proposed method is able to provide better or similar performance compare to methods, those use entire data patterns. The proposed method takes less computation time during training phase. 


\section{Simulation Results}

This paper compares Matlab based function called Generating fuzzy inference system (Genfis2) with the proposed model in terms of RMSE values which are calculated as follows

$$
R M S E=\sqrt{\frac{1}{n} \sum_{i=1}^{n}\left(y_{i}-\hat{y}_{i}\right)^{2}},
$$

where $n$ is the number of predictions, $y$ is the true value, and $y$ is the predicted value. The smaller RMSE is the better prediction by the proposed model.

\subsection{Nonlinear Dynamics System Identifi- cation Problem-I}

A nonlinear dynamics system identification problem is considered to illustrate the effect of the proposed method. The plant to be recognized is defined as

$$
y(t+1)=\frac{y(t) y(t-1)[y(t)+2.5]}{1+y^{2}(t)+y^{2}(t-1)}+u(t) .
$$

If a series-parallel identification model is used for recognizing the plant, the model can be defined as

$$
\hat{y}(t+1)=f\{y(t), y(t-1), u(t)\},
$$

where $y(t+1)$ is the output and $u(t)=\sin (2 \pi \mathrm{t} / 25)$ is the input; and this network includes three inputs and one output. The initial input values are considered as follows: $y(0)=0$ and $y(1)=0$. For each ${ }_{2}$ training and testing purpose, a set of 1000 data are extracted for this system identification problem.

Figures 10, 11 and 12 show the output surface of given fuzzy inference system (FIS) using the first two inputs and the output of given dataset. Figure 10 shows the output surface plot of FIS of Genfis2 by using the entire data patterns. Figures 11 and 12 show the output surface plot of FIS of the proposed method by using just a half of the data patterns for each dataset. Figures 11(a) and (b) show the surface plot, when dataset 1 collaborates with dataset 2 without any mapping of cluster center and with mapping of cluster center, respectively. Similarly, Figures 12 (a) and (b) show the surface plot when dataset 2 collaborates with dataset 1 without any mapping of cluster center and with mapping of cluster center, respectively.

\subsection{Nonlinear Dynamics System Identifi- cation Problem-II}

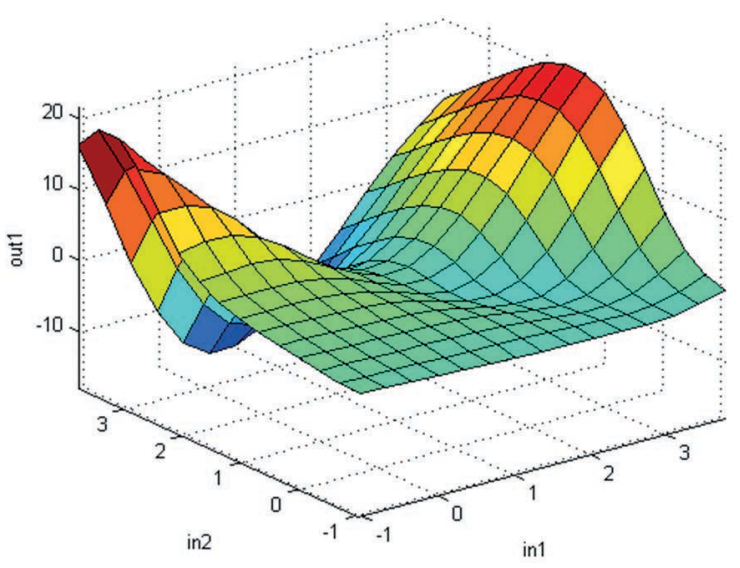

Figure 10. Surface plot of FIS for FCM on the entire data pattern

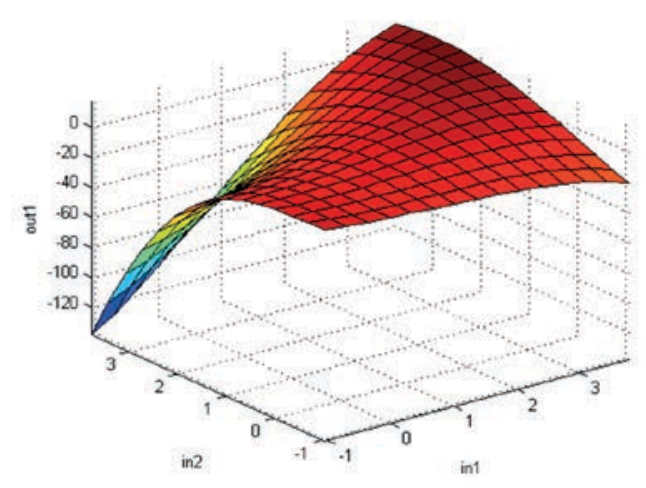

(a) Dataset 1 collaborates with dataset 2 without mapping

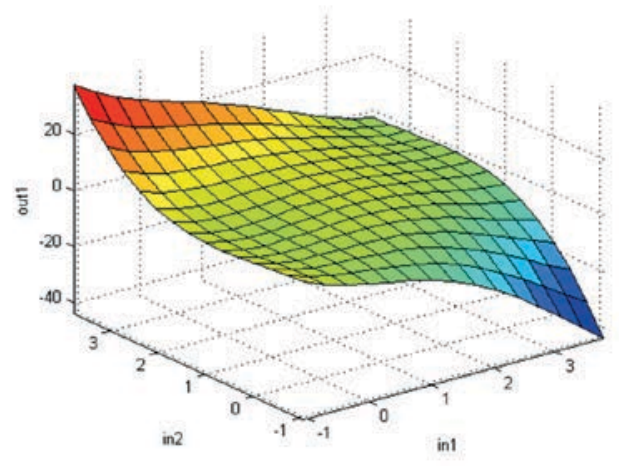

(b) Dataset 1 collaborates with dataset 2 with mapping

Figure 11. Surface plot of FIS for CFC and PCFC for $\beta=0.5$ 


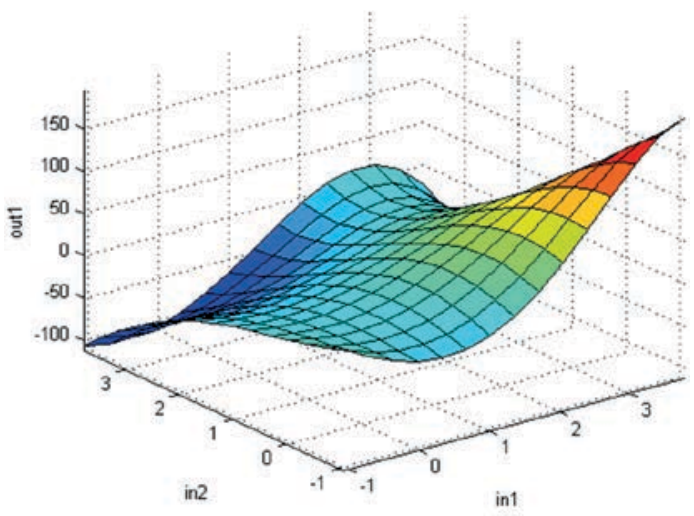

(a) Dataset2 collaborates with dataset 1 without mapping

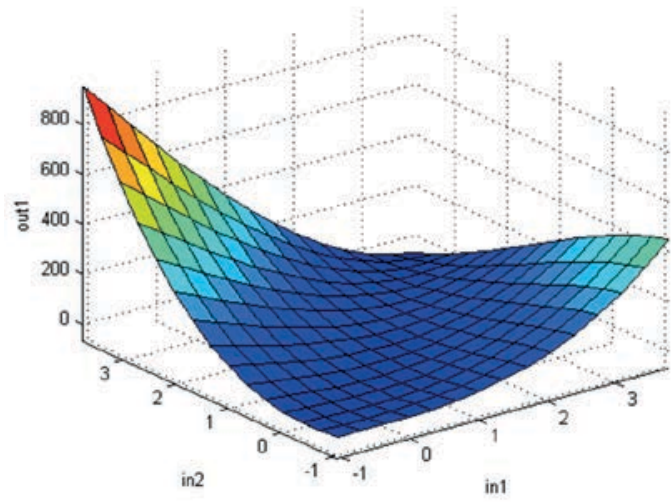

(b) Dataset 2 collaborates with dataset 1 with mapping

Figure 12. Surface plot of FIS for CFC and PCFC for $\beta=0.5$

The nonlinear system to be recognized is defined as

$$
y(t+1)=\frac{y(t)}{1+y^{2}(t)}+u^{3}(t)
$$

where $y(t+1)$ is the output and $u(t)$ is the input signal that is generated by using the sinusoidal function given by $u(t)=\sin (2 \pi \mathrm{t}) / 100$. The inputs $y(t)$ and $u(t)$ follow the uniform sample distribution in the interval $[-1.5,1.5]$ and $[-1.0,1.0]$, respectively.

For each, training and testing purpose, a set of 400 for each data patterns are generated, respectively. Further, training dataset is divided into two datasets: dataset 1 and dataset 2 , those contain 200 patterns each. The proposed method uses the knowledge representation of 200 patterns of dataset $1 /$ dataset 2 for network training after apply- ing the PCFC procedure. Table 1 shows the performance comparisons of the proposed method with different values of collaborative coefficient $(\beta)$ and Matlab based Genfis 2 model. When dataset 1 collaborates with dataset 2 , the best average training and testing RMSE value is 0.0068 and 0.0070 for $\beta=0.08$, respectively. When dataset 2 collaborates with dataset 1 , the best average training and testing RMSE value is 0.0071 and 0.0070 for $\beta=2$, respectively. While for Genfis 2 model, the training and testing RMSE value is 0.0134 and 0.0135 , respectively. Figures 13(a) and (b) show desired and predicted output during training and testing, respectively, at $\beta=1$ when dataset 1 collaborates with dataset 2 . Figure 14 shows desired and predicted output during training and testing, respectively, at $\beta=0.08$ when dataset 2 collaborates with dataset 1 .

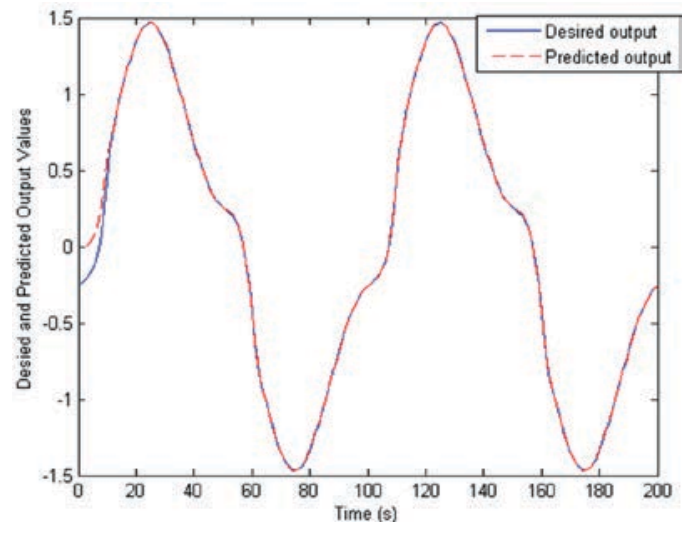

(a) Desired and predicted outputs during training

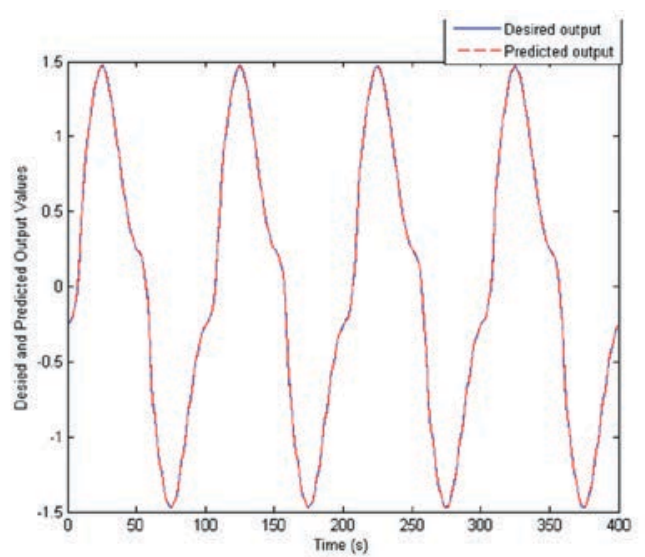

(b) Desired and predicted outputs during testing

Figure 13. Desired and predicted outputs during training and testing at $\beta=1$ when dataset 1 collaborates with dataset 2 


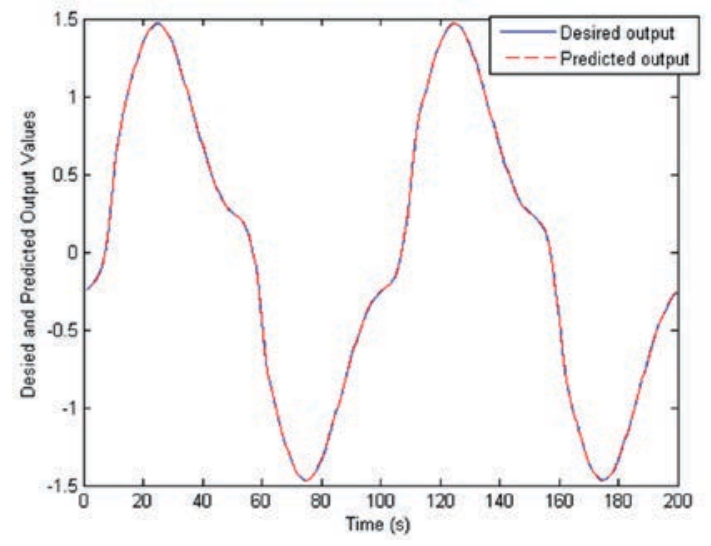

(a) Desired and predicted outputs during training

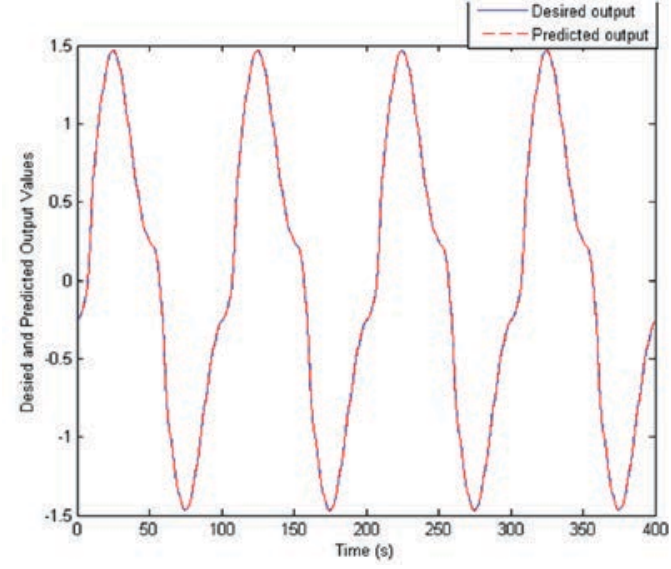

(b) Desired and predicted outputs during testing

Figure 14. Desired and predicted outputs during training and testing at $\beta=0.08$ when dataset 2 collaborates with dataset 1

Table 1. Training and testing RMSE of the proposed method and Genfis2

\begin{tabular}{|l|l|l|l|l|l|l|}
\hline Model & \multicolumn{2}{|l|}{$\begin{array}{l}\text { Proposed } \\
(\text { dataset1 } \rightarrow \text { dataset })\end{array}$} & $\begin{array}{l}\text { Method } \\
\text { Proposed } \\
\text { dataset2 } \rightarrow \text { dataset })\end{array}$ & \multicolumn{2}{|c|}{ Genfis2 } \\
\hline Process & $\begin{array}{l}\text { Training } \\
\text { RMSE }\end{array}$ & $\begin{array}{l}\text { Testing } \\
\text { RMSE }\end{array}$ & $\begin{array}{l}\text { Training } \\
\text { RMSE }\end{array}$ & $\begin{array}{l}\text { Testing } \\
\text { RMSE }\end{array}$ & $\begin{array}{l}\text { Training } \\
\text { RMSE }\end{array}$ & $\begin{array}{l}\text { Testing } \\
\text { RMSE }\end{array}$ \\
\hline$\beta$ & Mean & Mean & Mean & Mean & Mean & Mean \\
\hline 1 & $\mathbf{0 . 0 0 8 2}$ & $\mathbf{0 . 0 0 8 4}$ & 0.0079 & 0.0078 & 0.0134 & 0.0135 \\
\hline 2 & 0.0101 & 0.0103 & $\mathbf{0 . 0 0 7 1}$ & $\mathbf{0 . 0 0 7 0}$ & 0.0134 & 0.0135 \\
\hline 3 & 0.0084 & 0.0086 & 0.0072 & 0.0071 & 0.0134 & 0.0135 \\
\hline 4 & 0.0088 & 0.0089 & 0.0091 & 0.0091 & 0.0134 & 0.0135 \\
\hline 5 & 0.0097 & 0.0099 & 0.0096 & 0.0095 & 0.0134 & 0.0135 \\
\hline 6 & 0.0095 & 0.0097 & 0.0065 & 0.0065 & 0.0134 & 0.0135 \\
\hline 7 & 0.0106 & 0.0108 & 0.0090 & 0.0090 & 0.0134 & 0.0135 \\
\hline 8 & 0.0098 & 0.0100 & 0.0091 & 0.0091 & 0.0134 & 0.0135 \\
\hline 9 & 0.0093 & 0.0094 & 0.0079 & 0.0079 & 0.0134 & 0.0135 \\
\hline 10 & 0.0099 & 0.0101 & 0.0073 & 0.0073 & 0.0134 & 0.0135 \\
\hline 0.1 & 0.0084 & 0.0086 & 0.0088 & 0.0088 & 0.0134 & 0.0135 \\
\hline 0.2 & 0.0092 & 0.0094 & $\mathbf{0 . 0 0 7 7}$ & $\mathbf{0 . 0 0 7 7}$ & 0.0134 & 0.0135 \\
\hline 0.3 & 0.0085 & 0.0087 & 0.0092 & 0.0092 & 0.0134 & 0.0135 \\
\hline 0.4 & 0.0091 & 0.0093 & 0.0079 & 0.0079 & 0.0134 & 0.0135 \\
\hline 0.5 & 0.0103 & 0.0106 & 0.0087 & 0.0087 & 0.0134 & 0.0135 \\
\hline 0.6 & 0.0090 & 0.0092 & 0.0097 & 0.0097 & 0.0134 & 0.0135 \\
\hline 0.7 & 0.0082 & 0.0084 & 0.0087 & 0.0087 & 0.0134 & 0.0135 \\
\hline 0.07 & 0.0088 & 0.0090 & 0.0112 & 0.0112 & 0.0134 & 0.0135 \\
\hline 0.08 & $\mathbf{0 . 0 0 6 8}$ & $\mathbf{0 . 0 0 7 0}$ & 0.0095 & 0.0095 & 0.0134 & 0.0135 \\
\hline 0.09 & 0.0081 & 0.0083 & 0.0085 & 0.0085 & 0.0134 & 0.0135 \\
\hline
\end{tabular}




\section{Conclusions}

In this paper, we proposed a new modeling strategy for a TSK type fuzzy inference system based on collaborative fuzzy rule learning with the cluster center mapping technique. The proposed method helps system modeling to find an accurate model based on given input information by using knowledge representation of PCFC. The proposed method is able to provide better or similar performance while using just half of the given data patterns for training and keeping the lower computation time. For future work, we want to extend our work and compare with some other existing modeling systems with real world datasets and apply this model to deal with big data issue.

\section{Acknowledgment}

The authors would like to acknowledge the funding supported in part by Ministry of Science and Technology, R.O.C. under two Grant numbers: NSC102-2622-e-009-002-cc2 and MOST104-2218-e-009-003.

\section{References}

[1] E. H. Mamdani and S. Assilian, An experiment in linguistic synthesis with a fuzzy logic controller, International Journal of Man-machine Studies, vol. 7, pp. 1-13, 1975.

[2] T. Takagi and M. Sugeno, Fuzzy identification of systems and its applications to modeling and control, IEEE Transaction on System, Man, and Cybernetic, vol. 15, pp. 116-132, 1985.

[3] L. Rutkowski and K. Cpaka, Designing and learning of adjustable quasi-triangular norms with applications to neuro-fuzzy systems, IEEE Transaction on Fuzzy Systems, vol. 13, no. 1, pp. 140-151, 2005.

[4] L. Rutkowski and K. Cpaka, Flexible neuro-fuzzy systems, IEEE Transaction on Neural Networks, vol. 14 , no. 3, pp. 554-574, 2003.

[5] J. C. Bezdek, Pattern recognition with fuzzy objective function algorithms, Plenum Press, New York, 1981.

[6] J. C. Bezdek, R. Ehrlich, and W. Full, FCM: the fuzzy C-means clustering algorithm, Computers and Geosciences, vol. 10, no. 2-3, pp. 191-203, 1984.
[7] W. Pedrycz, Knowledge-based clustering: from data to information granules, A John Wiley \& Sons, Inc., Publication, 2005.

[8] W. Pedrycz, Collaborative fuzzy clustering, Pattern Recognition Letters, vol. 23, no. 14, pp. 1675-1686, 2002.

[9] W. Pedrycz and P. Rai, Collaborative Fuzzy Clustering with the use of Fuzzy C-Means and its Quantification, Fuzzy Sets and System, vol. 159, no. 18, pp. 2399-2427, 2008.

[10] C. T. Lin, M. Prasad, and J. Y Chang, Designing Mamdani Type Fuzzy Rule Using a Collaborative FCM Scheme, International Conference on Fuzzy Theory and Its Application, 2013.

[11] http://www.mathworks.com/help/fuzzy/genfis2.html

[12] R. Babuska, Fuzzy Modeling for Control, Norwell, MA: Kluwer, 1998.

[13] J. C. Dunn, A fuzzy relative of the ISODATA process and its use in detecting compact well separated clusters, Journal of Cybernetics, vol. 3, pp. 32-57, 1973.

[14] F. Hoppner, F. Klawonn, R. Kruse, and T. Runkler, Fuzzy Cluster Analysis: Methods for Classification. Data Analysis and Image Recognition, New York: Wiley, 1999.

[15] E. R. Hruschka, R. J. G. B. Campello, A. A. Freitas, and A. C. P. L. F. de Carvalho, A survey of evolutionary algorithms for clustering, IEEE Transaction on System Man Cybernetics- part-c, vol. 39, no. 2, pp. 133-155, 2009.

[16] R. Xu and D. Wunsch, Survey of clustering algorithms, IEEE Transaction on Neural Networks, vol. 16, no. 3, pp. 645-678, 2005.

[17] R. R. Yager and D. P. Filev, Approximate clustering via the mountain method, IEEE Transaction on System, Man, Cybernetics, vol. 24, no. 8, pp. 12791284,1994

[18] P. R. Kersten, Implementation issues in the fuzzy c-medians clustering algorithm, In Proceeding 6th IEEE International Conference on Fuzzy Systems, vol. 2, pp. 957-962, 1997.

[19] J. F. Kolen and T. Hutcheson, Reducing the time complexity of the fuzzy c-means algorithm, IEEE Transaction on Fuzzy Systems, vol. 10, no. 2, pp. 263-267, 2002.

[20] M. Sugeno and G. T. Kang, Structure identification of fuzzy model, Fuzzy Sets Systems, vol. 28, no. 1, pp. 15-33, 1988. 
[21] C. W. Ting and C. Quek, A Novel Blood Glucose Regulation Using - TSK FCMAC: A Fuzzy CMAC Based on the Zero-Ordered TSK Fuzzy Inference Scheme, IEEE Transaction on Neural Networks, vol. 20, no. 5, pp. 856-871, 2009.

[22] J. R. Castro, O. Castillo, P. Melin, and A. Rodrguez-Daz, A hybrid learning algorithm for a class of interval type-2 fuzzy neural networks, Information Science, vol. 179, no. 13, pp. 2175-2193, 2009.

[23] H. Song, C. Miao, Z. Shen, Y. Miao, and B. S. Lee, A fuzzy neural network with fuzzy impact grades, Neurocomputing, vol. 72, no. 13-15, pp. 3098-3122, 2009.

[24] D. Kim and C. Kim, Forecasting time series with genetic fuzzy predictor ensembles, IEEE Transaction on Fuzzy Systems, vol. 5, no. 4, pp. 523-535, 1997.

[25] M. Prasad, C. T. Lin, C. T. Yang and A. Saxena, Vertical Collaborative Fuzzy C-Means for Multiple
EEG Data Sets, Springer Lecture Notes in Computer Science, vol. 8102, pp. 246-257, 2013.

[26] R. N. Dave, and K. Bhaswan, Adaptive fuzzy cshells clustering and detection of ellipses, IEEE Transaction on Neural Networks, vol. 3, no. 5, pp. 643-662, 1992.

[27] Y. Man, and I. Gath, Detection and separation of ring-shaped clusters using fuzzy clustering, IEEE Transaction on Pattern Analysis and Machine Intelligence, vol. 16, no. 8, pp. 855-861, 1994.

[28] R. Krishnapuram, O. Nasraoui, and J. Keller, The fuzzy c spherical shells algorithm: a new approach, IEEE Transaction on Neural Networks, vol. 3, no. 5, pp. 663-671, 1992.

[29] M. Prasad, D. L. Li, Y. T. Liu, L. Siana, C. T. Lin, and A. Saxena, A Preprocessed Induced Partition Matrix Based Collaborative Fuzzy Clustering for Data Analysis, IEEE International Conference of Fuzzy Systems, pp. 1553-1558, 2014.

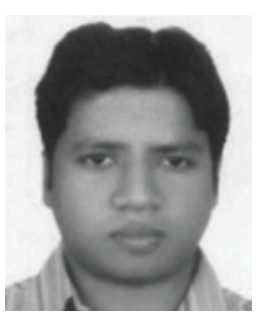

Mukesh Prasad received his Ph.D. degree in computer science from $\mathrm{Na}$ tional Chiao Tung University, Hsinchu, Taiwan in 2015 and master degree in computer application from Jawaharlal Nehru University, New Delhi, India, in 2009. He is currently associated with National Chiao Tung University, Hsinchu, Taiwan as Post-Doctoral Researcher. His current research interests include machine learning, pattern recognition, fuzzy systems, neural networks and Wireless Sensor Networks. Dr. Mukesh has published papers in international journal and conferences including IEEE Transactions, ACM, Elsevier and Springer.

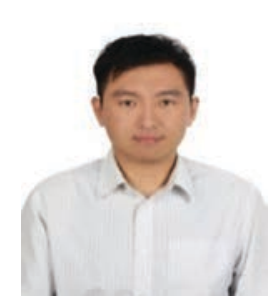

Yu-Ting Liu received the B.S. degree in Department of Electrical and Control Engineering from National ChiaoTung University, Hsinchu, Taiwan, in 2010 . He is currently pursuing the Ph.D. degree with the Institute of Electrical and Control Engineering, National Chiao Tung University, Hsinchu, Taiwan. His current research interests include fuzzy logic theory, machine learning, computer vision, EEG analysis and application.

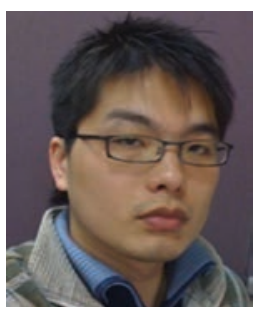

Dong-Lin Li received his Ph.D. degree in electrical engineering from National Chiao Tung University, Hsinchu, Taiwan in 2013 and master degree in electrical engineering from National Chung Hsing University, Taichung, Taiwan, in 2006. He is currently associated with National Chiao Tung University, Hsinchu, Taiwan as PostDoctoral Researcher. His current research interests include machine learning, fuzzy systems, neural networks and image processing and smart living technology. Dr. Li has published papers in international journal and conferences including IEEE Transactions, ACM, Elsevier and Springer.

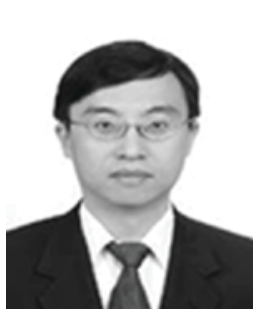

Dr. Chin-Teng Lin received the B.S. degree from National Chiao-Tung University (NCTU), Taiwan in 1986, and the Master and Ph.D. degree in electrical engineering from Purdue University, USA in 1989 and 1992, respectively. He is currently the Chair Professor of Faculty of Engineering and Information Technology, University of Technology Sydney, Chair Professor of Electrical and Computer Engineering, NCTU, International Faculty of University of California at San-Diego (UCSD), and Honorary Professorship of University of Nottingham. Dr. Lin was elevated to be an IEEE Fellow for his contributions to biologically inspired information systems in 2005, and was elevated International Fuzzy Systems Association (IFSA) Fellow in 2012. He is elected as the Editor-in-chief of IEEE Transactions on Fuzzy Systems since 2011. He also served on the Board of Governors at IEEE Circuits and Systems (CAS) Society in 2005-2008, IEEE Systems, Man, Cybernetics (SMC) Society in 2003-2005, IEEE Computational Intelligence Society (CIS) in 2008-2010, and Chair of IEEE Taipei Section in 2009-2010. He served as the Deputy Editor-in-Chief of IEEE Transactions on Circuits and Systems-II in 2006-2008. Dr. Lin is the coauthor of Neural Fuzzy Systems (Prentice-Hall), and the author of Neural Fuzzy Control Systems with Structure and Parameter Learning (World Scientific). He has published more than 200 journal papers (Total Citation: 20,155, H-index: 53, i10-index: 373) in the areas of neural networks, fuzzy systems, multimedia hardware/software, and cognitive neuro-engineering, including approximately 101 IEEE journal papers. 


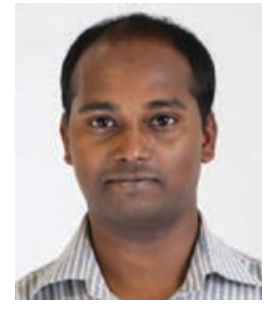

Rajiv Ratn Shah received the M.Tech. degree in computer technology and Applications from Department of Computer Science, Delhi Technolgical University, Delhi, India in 2010. Since January 2012 he is currently working towards the $\mathrm{PhD}$. degree from the School of Computing, National University of Singapore, Singapore. He worked as a Research Intern at the National Institute of Informatics, Japan, from November, 2015 to April, 2016. His research interests include the multimodal analysis of usergenerated content in the support of social media applications.

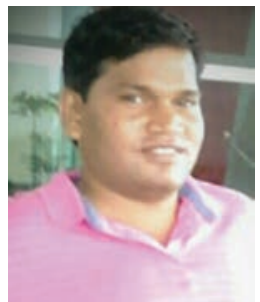

Omprakash Kaiwartya received Ph.D., M.Tech degrees in computer science from School of Computer and Systems Sciences, Jawaharlal Nehru University, New Delhi, India in 2015 and 2012 respectively. $\mathrm{He}$ is currently working as Post-Doctoral Fellow in Pervasive Computing Research Group, Faculty of Computing, Universiti Teknologi Malaysia (UTM), Johor Bahru, Malaysia. He served as area chair (Asia) in ICSNCS-2016. He is the reviewer of many reputed journals of communication field including IEEE sensors, IET Intelligent Transportation System, Adhoc Networks, etc. His research interests include Vehicular Ad-hoc Networks, Mobile Ad-hoc Networks and Wireless Sensor Networks. He is currently working in next generation vehicular networks known as 'Internet of Vehicles (IoV)'. Dr. Omprakash has published papers in reputed International Journals indexed in SCI and Conferences with publishers including ACM, IEEE, Springer, MDPI, KIIS, InderScience and Hindawi. 